\title{
Synchronization in Array of Coupled Neural Networks with Unbounded Distributed Delay and Limited Transmission Efficiency
}

\author{
Xinsong Yang, ${ }^{1}$ Mengzhe Zhou, ${ }^{2}$ and Jinde $\mathrm{Cao}^{2,3}$ \\ ${ }^{1}$ Department of Mathematics, Chongqing Normal University, Chongqing 400047, China \\ ${ }^{2}$ Department of Mathematics, Southeast University, Nanjing 210096, China \\ ${ }^{3}$ Department of Mathematics, King Abdulaziz University, Jeddah 21589, Saudi Arabia \\ Correspondence should be addressed to Jinde Cao; jdcao@seu.edu.cn
}

Received 25 January 2013; Accepted 11 March 2013

Academic Editor: Qi Luo

Copyright ( 2013 Xinsong Yang et al. This is an open access article distributed under the Creative Commons Attribution License, which permits unrestricted use, distribution, and reproduction in any medium, provided the original work is properly cited.

\begin{abstract}
This paper investigates global synchronization in an array of coupled neural networks with time-varying delays and unbounded distributed delays. In the coupled neural networks, limited transmission efficiency between coupled nodes, which makes the model more practical, is considered. Based on a novel integral inequality and the Lyapunov functional method, sufficient synchronization criteria are derived. The derived synchronization criteria are formulated by linear matrix inequalities (LMIs) and can be easily verified by using Matlab LMI Toolbox. It is displayed that, when some of the transmission efficiencies are limited, the dynamics of the synchronized state are different from those of the isolated node. Furthermore, the transmission efficiency and inner coupling matrices between nodes play important roles in the final synchronized state. The derivative of the time-varying delay can be any given value, and the time-varying delay can be unbounded. The outer-coupling matrices can be symmetric or asymmetric. Numerical simulations are finally given to demonstrate the effectiveness of the theoretical results.
\end{abstract}

\section{Introduction}

In the past few decades, the problem of chaos synchronization and network synchronization has been extensively studied since its potential engineering applications such as communication, biological systems, and information processing (see [1-4] and the references therein). It is found out that neural networks can exhibit chaotic behavior as long as their parameters and delays are properly chosen [5]. Recently, synchronization of coupled chaotic neural networks has received much attention due to its wide applications in many areas [612].

An array of coupled neural networks, as a special class of complex networks [12-16], has received increasing attention from researchers of different disciplines. In the literature, synchronization in an array of coupled neural networks has been extensively studied $[8,17-20]$. The authors of [8] studied the exponential synchronization problem for coupled neural networks with constant time delay and stochastic noise perturbations. Some novel $H_{\infty}$ synchronization results have been obtained in [21] for a class of discrete timevarying stochastic networks over a finite horizon. In [18-20, 22], several types of synchronization in dynamical networks with discrete and bounded distributed delays were studied based on LMI approach. However, most of the obtained results concerning synchronization of complex networks including the above-mentioned implicitly assume that the connections among nodes can transmit information from the dispatcher nodes to receiver ones according to the expected effect. In other words, the transmission efficiencies between connected nodes are all perfect. In practical situations, signal transmission efficiency between nodes is limited in general due to either the limited bandwidth of the channels or external causes such as uncertain noisy perturbations and artificial factors. If the transmission efficiency of some connections in a complex network is limited, then most of the existing synchronization criteria are not applicable. Consequently, it is urgent to propose new synchronization 
criteria for complex networks with arbitrary transmission efficiency.

Time delays usually exist in neural networks. Some papers concerning synchronization of neural networks have considered various time delays. In [6], Cao and $\mathrm{Lu}$ investigated the adaptive synchronization of neural networks with or without time-varying delay. In [23], synchronization of neural networks with discrete and bounded distributed time-varying delays was investigated. The authors of [8] studied the exponential synchronization problem for coupled neural networks with constant time delay. Synchronization of coupled neural networks with both discrete and bounded distributed delays was studied in $[11,18-20,24]$. As pointed out in [25], bounded distributed delay means that there is a distribution of propagation delays only over a period of time. At the same time, unbounded distributed delay implies that the distant past has less influence compared to the recent behavior of the state [26]. Note that most existing results on stability or synchronization of neural networks with bounded distributed delays obtained by using LMI approach cannot be directly extended to those with unbounded distributed delays. Although there were some results on stability or synchronization of neural networks with unbounded distributed delays, some of them were obtained by using algebra approach [27-30]. As is well known, compared with LMI result, algebraic one is more conservative, and criteria in terms of LMI can be easily checked by using the powerful Matlab LMI Toolbox. Therefore, in this paper we investigate the synchronization in an array of coupled neural networks with both discrete time-varying delays and unbounded distributed delays based on LMI approach. Results of the present paper are also applicable to synchronization of complex networks with bounded or unbounded distributed time delay.

Motivated by the above analysis, this paper studies the synchronization in an array neural network with both timevarying delays and unbounded distributed delays, under the condition that the transmission efficiencies among nodes are limited. By using a new lemma on infinite integral inequality and the Lyanupov functional method, some synchronization criteria formulated by LMIs are obtained for the considered model. In the obtained synchronization criteria, the timevarying delay studied can be unbounded, and its derivative can be any given value. Especially, when some of the transmission efficiencies are limited (i.e., less than 1), the transmission efficiency and inner coupling matrices between nodes have serious impact on the synchronized state. Results of this paper extend some existing ones. Numerical simulations are finally given to demonstrate the effectiveness of the theoretical results.

The rest of this paper is organized as follows. In Section 2, coupled neural network model with transmission efficiency is presented. Some lemmas and necessary assumptions are also given in this section. Synchronization criteria of the considered model are obtained in Section 3. Then, in Section 4, numerical simulations are given to show the effectiveness of our results. Finally, Section 5 reaches conclusions.

Notations. In the sequel, if not explicitly stated, matrices are assumed to have compatible dimensions. $I_{q}$ denotes the identity matrix of $q$-dimension. For vector $x \in \mathbb{R}^{n}$, the norm is denoted as $\|x\|=\sqrt{x^{T} x}$, where $T$ denotes transposition. $A=\left(a_{i j}\right)_{m \times m}$ denotes a matrix of $m \times m$-dimension. $A>0$ or $A<0$ denotes that the matrix $A$ is a symmetric and positive or negative definite matrix.

\section{Preliminaries}

An array of coupled neural networks consisting of $N$ identical nodes with delays and transmission efficiencies is described as follows:

$$
\begin{aligned}
& \dot{x}_{i}(t)=-C x_{i}(t)+A f\left(x_{i}(t)\right)+B f\left(x_{i}(t-\tau(t))\right) \\
&+D \int_{-\infty}^{t} K(t-s) f\left(x_{i}(s)\right) \mathrm{d} s+I(t) \\
&+\sum_{j=1}^{N} \alpha_{i j} u_{i j} \Phi x_{j}(t)+\sum_{j=1}^{N} \beta_{i j} v_{i j} \Upsilon x_{j}(t-\tau(t)) \\
&+\sum_{j=1}^{N} \gamma_{i j} w_{i j} \Lambda \int_{-\infty}^{t} K(t-s) x_{j}(s) \mathrm{d} s, \\
& i=1,2, \ldots, N,
\end{aligned}
$$

where $x_{i}(t)=\left(x_{i 1}(t), \ldots, x_{i n}(t)\right)^{T} \in \mathbb{R}^{n}$ represents the state vector of the $i$ th node of the network at time $t ; n$ corresponds to the number of neurons; $f\left(x_{i}(t)\right)=\left(f_{1}\left(x_{i 1}(t)\right), \ldots\right.$, $\left.f_{n}\left(x_{i n}(t)\right)\right)^{T}$ is the neuron activation function; $C=\operatorname{diag}\left(c_{1}\right.$, $\left.c_{2}, \ldots, c_{n}\right)$ is a diagonal matrix with $c_{i}>0 ; A=\left(a_{i j}\right)_{n \times n}, B=$ $\left(b_{i j}\right)_{n \times n}$, and $D=\left(d_{i j}\right)_{n \times n}$ are the connection weight matrix, time-delayed weight matrix, and the distributively timedelayed weight matrix, respectively; $I(t)=\left(I_{1}(t), I_{2}(t), \ldots\right.$, $\left.I_{n}(t)\right)^{T} \in \mathbb{R}^{n}$ is an external input vector; $\tau(t)$ denotes the time-varying delay satisfying $\dot{\tau}(t) \leq h, h$ is a constant; $K(\cdot)$ is a scalar function describing the delay kernel. The $\Phi=$ $\left(\phi_{i j}\right)_{n \times n}, \Upsilon=\left(\varepsilon_{i j}\right)_{n \times n}$, and $\Lambda=\left(\lambda_{i j}\right)_{n \times n}$ are inner coupling matrices of the networks, which describe the individual coupling between two subsystems. Matrices $U=\left(u_{i j}\right)_{N \times N}, V=$ $\left(v_{i j}\right)_{N \times N}$, and $W=\left(w_{i j}\right)_{N \times N}$ are outer couplings of the whole networks satisfying the following diffusive conditions:

$$
\begin{array}{ll}
u_{i j} \geq 0(i \neq j), & u_{i i}=-\sum_{j=1, j \neq i}^{N} u_{i j}, \quad i, j=1,2, \ldots, N, \\
v_{i j} \geq 0(i \neq j), & v_{i i}=-\sum_{j=1, j \neq i}^{N} u_{i j}, \quad i, j=1,2, \ldots, N, \\
w_{i j} \geq 0(i \neq j), & w_{i i}=-\sum_{j=1, j \neq i}^{N} w_{i j}, \quad i, j=1,2, \ldots, N .
\end{array}
$$

Matrices $\alpha=\left(\alpha_{i j}\right)_{N \times N}, \beta=\left(\beta_{i j}\right)_{N \times N}$, and $\Gamma=\left(\gamma_{i j}\right)_{N \times N}$ are transmission efficiency matrices of the coupled network. The constants $0 \leq \alpha_{i j}, \beta_{i j}, \gamma_{i j} \leq 1$ represent, respectively, signal transmission efficiency from node $j$ to node $i$ through connections $u_{i j}$, $v_{i j}$, and $w_{i j}$. In this paper, we always assume that

$$
\alpha_{i i}=\beta_{i i}=\gamma_{i i}=1, \quad i=1,2, \ldots, N .
$$

The initial condition of (1) is given by $x_{i}(t)=\phi_{i}(t) \epsilon$ $C\left([-\infty, 0], \mathbb{R}^{n}\right), i=1,2, \ldots, N$. In this paper, we assume that 
at least one matrix of $U, V$, and $W$ is irreducible in the sense that there is no isolated node in corresponding graph.

Remark 1. Model (1) is general, and some special models can be derived from it. For instance, if

$$
K(s)= \begin{cases}0, & s>\theta(t), \\ K(s), & 0 \leq s \leq \theta(t),\end{cases}
$$

for any scalar $\theta(t)>0, t \in \mathbb{R}$, then the network (1) becomes the following coupled neural network with bounded distributed delays and transmission efficiencies:

$$
\begin{aligned}
& \dot{x}_{i}(t)=-C x_{i}(t)+A f\left(x_{i}(t)\right)+B f\left(x_{i}(t-\tau(t))\right) \\
&+D \int_{t-\theta(t)}^{t} K(t-s) f\left(x_{i}(s)\right) \mathrm{d} s+I(t) \\
&+\sum_{j=1}^{N} \alpha_{i j} u_{i j} \Phi x_{j}(t)+\sum_{j=1}^{N} \beta_{i j} v_{i j} \Upsilon x_{j}(t-\tau(t)) \\
&+\sum_{j=1}^{N} \gamma_{i j} w_{i j} \Lambda \int_{t-\theta(t)}^{t} K(t-s) x_{j}(s) \mathrm{d} s, \\
& i=1,2, \ldots, N,
\end{aligned}
$$

which includes the models in $[18,19]$ as a special case when $\tau(t)=\tau, \theta(t)=\theta$, and $\alpha_{i j}=\beta_{i j}=\gamma_{i j}=1,1 \leq i, j \leq N$, where $\tau, \theta$ are nonnegative constants. Furthermore, if $K(s)=1,0 \leq$ $s \leq \theta$, then (1) turns out to the model studied in [20].

Remark 2. We introduce transmission efficiencies between nodes in model (1). The two extreme situations are if all the signal channels in the network operate perfectly, then $\alpha_{i j}=$ $\beta_{i j}=\gamma_{i j}=1,1 \leq i, j \leq N$; if no signal is transmitted through $u_{i j}, v_{i j}$, and $w_{i j}$ or $u_{i j}=v_{i j}=w_{i j}=0, i \neq j$, then $\alpha_{i j}=\beta_{i j}=$ $\gamma_{i j}=0, i \neq j$. Since many practical factors such as limited bandwidth of the channels or external causes and other uncertain perturbations surely exist, the model (1) is more practical than existing models of complex networks including those in [18-20].

Based on (2)-(3), the system (1) can be written as

$$
\begin{aligned}
\dot{x}_{i}(t)= & -C x_{i}(t)+A f\left(x_{i}(t)\right)+B f\left(x_{i}(t-\tau(t))\right) \\
& +D \int_{-\infty}^{t} K(t-s) f\left(x_{i}(s)\right) \mathrm{d} s+I(t) \\
& +\sum_{j=1}^{N} \bar{u}_{i j} \Phi x_{j}(t)+\sum_{j=1}^{N} \bar{v}_{i j} \Upsilon x_{j}(t-\tau(t)) \\
& +\sum_{j=1}^{N} \bar{w}_{i j} \Lambda \int_{-\infty}^{t} K(t-s) x_{j}(s) \mathrm{d} s \\
& -\sum_{j=1, j \neq i}^{N}\left(1-\alpha_{i j}\right) u_{i j} \Phi x_{i}(t) \\
& -\sum_{j=1, j \neq i}^{N}\left(1-\beta_{i j}\right) v_{i j} \Upsilon x_{i}(t-\tau(t)) \\
& -\sum_{j=1, j \neq i}^{N}\left(1-\gamma_{i j}\right) w_{i j} \Lambda \int_{-\infty}^{t} K(t-s) x_{i}(s) \mathrm{d} s,
\end{aligned}
$$

where $\bar{u}_{i j}=\alpha_{i j} u_{i j}, \bar{u}_{i i}=-\sum_{j=1, j \neq i} \alpha_{i j} u_{i j}, \bar{v}_{i j}=\beta_{i j} v_{i j}, \bar{v}_{i i}=$ $-\sum_{j=1, j \neq i} \beta_{i j} v_{i j}, \bar{w}_{i j}=\gamma_{i j} w_{i j}$, and $\bar{w}_{i i}=-\sum_{j=1, j \neq i} \gamma_{i j} w_{i j}, i \neq j$. Obviously, the matrices $\bar{U}=\left(\bar{u}_{i j}\right)_{N \times N}, \bar{V}=\left(\bar{v}_{i j}\right)_{N \times N}$ and $\bar{W}=$ $\left(\bar{w}_{i j \infty}\right)_{N \times N}$ are diffusive.

This paper utilizes the following assumptions.

$\left(\mathrm{H}_{1}\right)$ The delay kernel $K:[0,+\infty) \rightarrow[0,+\infty)$ is a realvalued nonnegative continuous function, and there exists positive number $k$ such that $\int_{0}^{+\infty} K(s) \mathrm{d} s=k$.

$\left(\mathrm{H}_{2}\right)$ There exist constant matrices $E_{1}$ and $E_{2}$ such that

$$
\begin{aligned}
& {\left[f(x)-f(y)-E_{1}(x-y)\right]^{T}} \\
& \quad \times\left[f(x)-f(y)-E_{2}(x-y)\right] \leq 0, \quad \forall x, y \in \mathbb{R}^{n} .
\end{aligned}
$$

$\left(\mathrm{H}_{3}\right)$ There are constants $a, b, c$ such that $\sum_{j=1, j \neq i}^{N}(1-$ $\left.\alpha_{i j}\right) u_{i j}=a, \sum_{j=1, j \neq i}^{N}\left(1-\beta_{i j}\right) v_{i j}=b$, and $\sum_{j=1, j \neq i}^{N}(1-$ $\left.\gamma_{i j}\right) w_{i j}=c, i=1,2, \ldots, N$.

Remark 3. The assumption $\left(\mathrm{H}_{2}\right)$ was used in $[24,31] . f$ satisfies the sector condition in the sense that belongs to the sectors $\left[E_{1}, E_{2}\right]$. Such a sector description is quit general and includes the usual Lipschitz conditions as a special case.

Remark 4. When the transmission efficiencies of all the channels are considered and some of them are limited, the final synchronized state is different from that of a single node without coupling. According to $\left(\mathrm{H}_{3}\right)$, the synchronized state can be described as the following:

$$
\begin{aligned}
\dot{z}(t)= & -(C+a \Phi) z(t)+A f(z(t))+B f(z(t-\tau(t))) \\
& +D \int_{-\infty}^{t} K(t-s) f(z(s)) \mathrm{d} s+I(t) \\
& -b \Upsilon z(t-\tau(t))-c \Lambda \int_{-\infty}^{t} K(t-s) z(s) \mathrm{d} s .
\end{aligned}
$$

In order to derive our main results, some basic definitions and useful lemmas are needed.

Definition 5. The coupled neural network with limited transmission efficiency (1) is said to be globally asymptotically synchronized if

$$
\lim _{t \rightarrow \infty}\left\|x_{i}(t)-x_{j}(t)\right\|=0, \quad i=1,2, \ldots, N,
$$

holds for any initial values.

Lemma 6 (see [32]). Let $\otimes$ denote the Kronecker product, $A, B, C$, and $D$ are matrices with appropriate dimensions. The following properties are satisfied:

(1) $(a A) \otimes B=A \otimes(a B)$, where $a$ is a constant;

(2) $(A+B) \otimes C=A \otimes C+B \otimes C$;

(3) $(A \otimes B)(C \otimes D)=(A C) \otimes B D$.

Let $T(\epsilon)$ denote the set of matrices of which the sum of the element in each row is equal to the real number $\epsilon$. The set $M_{1}$ 
is defined as follows: if $M=\left(M_{i j}\right)_{(N-1) \times N} \in M_{1}$, each row of $M$ contains exactly one element 1 and one element -1 , and all other elements are zero. $j_{i 1}\left(j_{i 2}\right)$ denotes the column indexes of the first (second) nonzero element in the $i$ th row. The set $H$ is defined by $H=\left\{\left\{j_{11}, j_{12}\right\},\left\{j_{21}, j_{22}\right\}, \ldots,\left\{j_{p 1}, j_{p 2}\right\}\right\}$. The set $M_{2}$ is defined as follows: $M_{2} \subset M_{1}$ and if $M=\left(m_{i j}\right)_{(N-1) \times N} \in$ $M_{2}$, for any pair of the column indexes $j_{s}$ and $j_{t}$, there exist indexes $j_{1}, j_{2}, \ldots, j_{l}$ with $j_{1}=j_{s}$ and $j_{l}=j_{t}$ such that $\left\{j_{m}, j_{m+1}\right\} \in H$ for $m=1,2, \ldots, l-1$.

Lemma 7 (see $[33,34])$. Let $M \in M_{2}$ be $a(N-1) \times N$ matrix and $G \in T(\epsilon)$ be a $N \times N$ matrix. Then, there exists a $N \times(N-1)$ matrix $J$ such that $M G=\widetilde{G} M$, where $\widetilde{G}=M G J$. Moreover, let $\Phi$ be a constant $n \times n$ matrix and $\mathrm{G}=G \otimes \Phi$, then $\mathrm{MG}=\widetilde{\mathrm{G}} \mathrm{M}$, where $\widetilde{\mathrm{G}}=\widetilde{\mathrm{G}} \otimes \Phi, \mathrm{M}=M \otimes I_{n}$. Furthermore, $M J=I_{N-1}$.

The following lemma can be easily obtained from $[18,33]$.

Lemma 8. Let $x(t)=\left(x_{1}^{T}(t), x_{1}^{T}(t), \ldots, x_{N}^{T}(t)\right)^{T}$ and $M \in M_{2}$, if $\lim _{t \rightarrow \infty}\left\|\left(M \otimes I_{n}\right) x(t)\right\|=0$, then $\lim _{t \rightarrow \infty}\left\|x_{i}(t)-x_{j}(t)\right\|=$ 0 , for all $i, j=1,2, \ldots, N$.

Lemma 9 (see [35]). Suppose $K(t)$ is a nonnegative bounded scalar function defined on $[0,+\infty)$ and $\int_{0}^{+\infty} K(u) d u=k$. For any constant matrix $D \in \mathbb{R}^{n \times n}, D>0$, and vector function $x:(-\infty, t] \rightarrow \mathbb{R}^{n}$ for $t \geq 0$, one has

$$
\begin{aligned}
& k \int_{-\infty}^{t} K(t-s) x^{T}(s) D x(s) \mathrm{d} s \\
& \quad \geq\left(\int_{-\infty}^{t} K(t-s) x(s) \mathrm{d} s\right)^{T} D \int_{-\infty}^{t} K(t-s) x(s) \mathrm{d} s .
\end{aligned}
$$

Provided that the integrals are all well defined.

\section{Synchronization with Limited Transmission Efficiency}

In this section, synchronization criteria formulated by LMIs of the general model (1) are derived. When the distributed delays in (1) are bounded, corresponding synchronization criterion is also obtained. In the derived synchronization criteria, the time-varying delays can be unbounded and their derivative can be any given value.

For $M \in M_{2}$, by Lemma 7, there exists a $N \times(N-1)$ matrix $J$ such that $M J=I_{N-1}$. Let $\bar{U}=\bar{U} \otimes \Phi, \widetilde{U}=\widetilde{U} \otimes \Phi$, $\widetilde{U}=M \bar{U} J, \bar{V}=\bar{V} \otimes \Upsilon, \widetilde{V}=\widetilde{V} \otimes \Upsilon, \widetilde{V}=M \bar{V} J, \overline{\mathrm{W}}=$ $\bar{W} \otimes \Lambda, \widetilde{W}=\widetilde{W} \otimes \Lambda, \widetilde{W}=M \bar{W} J, \bar{C}=C+a \Phi, \bar{C}=I_{N} \otimes \bar{C}$, $\overline{\mathrm{C}}_{1}=I_{N-1} \otimes \bar{C}, \mathrm{~A}=I_{N} \otimes A, \mathrm{~A}_{1}=I_{N-1} \otimes A, \mathrm{~B}=I_{N} \otimes B$, $\mathrm{B}_{1}=I_{N-1} \otimes B, \mathrm{D}=I_{N} \otimes D, \mathrm{D}_{1}=I_{N-1} \otimes D, \mathrm{~K}=I_{N} \otimes K$, $\mathrm{K}_{1}=I_{N-1} \otimes K, \mathrm{f}(x(t))=\left(f\left(x_{1}(t)\right), f\left(x_{2}(t)\right), \ldots, f\left(x_{N}(t)\right)\right)^{T}$, $\mathrm{I}(\mathrm{t})=(I(t), I(t), \ldots, I(t))^{T}, \bar{\Upsilon}=I_{N} \otimes b \Upsilon, \bar{\Upsilon}_{1}=I_{N-1} \otimes b \Upsilon, \bar{\Lambda}=$ $I_{N} \otimes c \Lambda, \bar{\Lambda}_{1}=I_{N-1} \otimes c \Lambda, x_{i}(t)=\left(x_{i 1}(t), x_{i 2}(t), \ldots, x_{i n}(t)\right)^{T}$, $x(t)=\left(x_{1}(t), x_{2}(t), \ldots, x_{N}(t)\right)^{T}$. Then, the network (1) can be written in the Kronecker product form as

$$
\begin{aligned}
\dot{x}(t)= & -\overline{\mathrm{C}} x(t)+\mathrm{Af}(x(t))+\mathrm{Bf}(x(t-\tau(t))) \\
& +\mathrm{D} \int_{-\infty}^{t} \mathrm{~K}(t-s) \mathrm{f}(x(s)) \mathrm{d} s+\mathrm{I}(t) \\
& +\overline{\mathrm{U}} x(t)+\overline{\mathrm{V}} x(t-\tau(t)) \\
& +\overline{\mathrm{W}} \int_{-\infty}^{t} \mathrm{~K}(t-s) x(s) \mathrm{d} s-\bar{\Upsilon} x(t-\tau(t)) \\
& -\bar{\Lambda} \int_{-\infty}^{t} \mathrm{~K}(t-s) x(s) \mathrm{d} s .
\end{aligned}
$$

To obtain synchronization criterion in the array of coupled neural networks (1), we only need to consider the the problem for the system (11). Theorem 10 is our main result.

Theorem 10. Under assumptions $\left(H_{1}\right)-\left(H_{3}\right)$, if there exist matrices $M \in M_{2}$ and $J$ satisfying $M J=I_{N-1}$, positive definite matrices $P, Q, R, G, S \in \mathbb{R}^{(N-1) n \times(N-1) n}$ and two positive diagonal matrices $S_{1}, S_{2} \in \mathbb{R}^{(N-1) n \times(N-1) n}$ such that

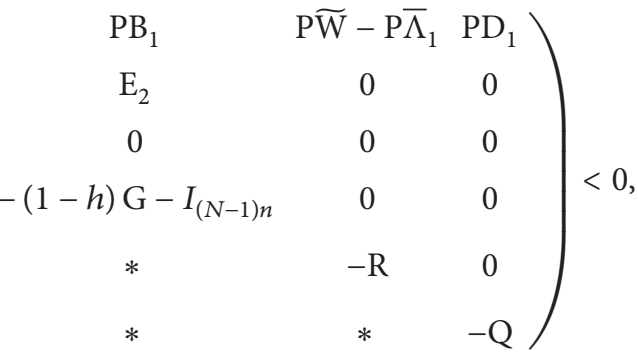

Proof. Consider the following Lyapunov function:

$$
V(t)=\sum_{i=1}^{5} V_{i}(t),
$$
synchronized. 
where

$$
\begin{aligned}
& V_{1}(t)= x^{T}(t) \mathrm{M}^{T} \operatorname{PM} x(t), \\
& V_{2}(t)=k \int_{-\infty}^{0} \int_{t+s}^{t}(\mathrm{MK}(t-\theta) \mathrm{f}(x(\theta)))^{T} \\
& \times \mathrm{Q}(\mathrm{MK}(t-\theta) \mathrm{f}(x(\theta))) \mathrm{d} \theta \mathrm{d} s, \\
& V_{3}(t)=k \int_{-\infty}^{0} \int_{t+s}^{t}(\mathrm{MK}(t-\theta) x(\theta))^{T} \\
& \quad \times \mathrm{R}(\mathrm{MK}(t-\theta) x(\theta)) \mathrm{d} \theta \mathrm{d} s, \\
& V_{4}(t)=\int_{t-\tau(t)}^{t}(\operatorname{Mf}(x(s)))^{T} \mathrm{G}(\mathrm{Mf}(x(s))) \mathrm{d} s, \\
& \dot{V}_{5}(t)=(\operatorname{Mr} x(t))^{T} \mathrm{~S}(\mathrm{M} x(t)) \\
&-(1-\dot{\tau}(t))(\mathrm{M} x(t-\tau(t)))^{T} \mathrm{~S}(\mathrm{M} x(t-\tau(t))) .
\end{aligned}
$$

Differentiating $V_{1}(t)$ along the solution of (11) obtains that

$$
\begin{aligned}
\dot{V}_{1}(t)=-x^{T}(t)\left(\overline{\mathrm{C}}^{T} \mathrm{M}^{T} \mathrm{PM}\right. & \left.+\mathrm{M}^{T} \mathrm{PM} \overline{\mathrm{C}}\right) x(t) \\
+2 x^{T}(t) \mathrm{M}^{T} \mathrm{PM}[\mathrm{Af}(x(t))+\mathrm{Bf}(x(t-\tau(t))) & \\
& +\mathrm{D} \int_{-\infty}^{t} \mathrm{~K}(t-s) \mathrm{f}(x(s)) \mathrm{d} s \\
& +\mathrm{I}(t)+\overline{\mathrm{U}} x(t)+\overline{\mathrm{V}} x(t-\tau(t)) \\
& +\overline{\mathrm{W}} \int_{-\infty}^{t} \mathrm{~K}(t-s) x(s) \mathrm{d} s \\
& -\bar{\Upsilon} x(t-\tau(t)) \\
& \left.-\bar{\Lambda} \int_{-\infty}^{t} \mathrm{~K}(t-s) x(s) \mathrm{d} s\right]
\end{aligned}
$$

By virtue of Lemma 6 , it can be verified that $M \bar{C}=\bar{C}_{1} M$, $\mathrm{MA}=\mathrm{A}_{1} \mathrm{M}, \mathrm{MB}=\mathrm{B}_{1} \mathrm{M}, \mathrm{MD}=\mathrm{D}_{1} \mathrm{M}, \mathrm{MK}=\mathrm{K}_{1} \mathrm{M}$, $\mathrm{M} \bar{\Upsilon}=\bar{\Upsilon}_{1} \mathrm{M}, \mathrm{M} \bar{\Lambda}=\bar{\Lambda}_{1} \mathrm{M}$, and $\mathrm{MI}(t)=0$. On the other hand, it follows from Lemma 7 that $M \bar{U}=\widetilde{U} M, M \bar{V}=\widetilde{V} M$, and $\mathrm{MW}=\widetilde{\mathrm{W}} \mathrm{M}$. Therefore,

$$
\begin{aligned}
\dot{V}_{1}(t)=-x^{T}(t)\left(\mathrm{M}^{T} \overline{\mathrm{C}}_{1}^{\mathrm{T}} \mathrm{PM}+\mathrm{M}^{T} \overline{\mathrm{PC}}_{1} \mathrm{M}\right) x(t) \\
+2 x^{T}(t) \mathrm{M}^{T} \mathrm{P}\left[\mathrm{A}_{1} \mathrm{Mf}(x(t))\right. \\
+\mathrm{B}_{1} \mathrm{Mf}(x(t-\tau(t))) \\
+\mathrm{D}_{1} \mathrm{M} \int_{-\infty}^{t} \mathrm{~K}(t-s) \mathrm{f}(x(s)) \mathrm{d} s \\
+\widetilde{\mathrm{UM}} x(t)+\widetilde{\mathrm{V} M} x(t-\tau(t)) \\
+\widetilde{\mathrm{W} M} \int_{-\infty}^{t} \mathrm{~K}(t-s) x(s) \mathrm{d} s
\end{aligned}
$$

$$
\begin{aligned}
& -\bar{\Upsilon}_{1} \mathrm{M} x(t-\tau(t)) \\
& \left.-\bar{\Lambda}_{1} \mathrm{M} \int_{-\infty}^{t} \mathrm{~K}(t-s) x(s) \mathrm{d} s\right] .
\end{aligned}
$$

Moreover, based on Lemma 9, one gets that

$$
\begin{gathered}
\dot{V}_{2}(t) \leq k^{2}(\operatorname{MK}(0) \mathrm{f}(x(t)))^{T} \mathrm{Q}(\operatorname{MK}(0) \mathrm{f}(x(t))) \\
-k \int_{-\infty}^{t}(\operatorname{MK}(t-s) \mathrm{f}(x(s)))^{T} \\
\times \mathrm{Q}(\operatorname{MK}(t-s) \mathrm{f}(x(s))) \mathrm{d} s \\
\leq k^{2}(\operatorname{Mf}(x(t)))^{T} \mathrm{~K}^{T}(0) \mathrm{QK}(0)(\operatorname{Mf}(x(t))) \\
-\left(\int_{-\infty}^{t} \operatorname{MK}(t-s) \mathrm{f}(x(s)) \mathrm{d} s\right)^{T} \\
\times \mathrm{Q}\left(\int_{-\infty}^{t} \operatorname{MK}(t-s) \mathrm{f}(x(s)) \mathrm{d} s\right) .
\end{gathered}
$$

Similarly,

$$
\begin{aligned}
\dot{V}_{3}(t) \leq & k^{2}(\mathrm{M} x(t))^{T} \mathrm{~K}^{T}(0) \mathrm{RK}(0)(\mathrm{M} x(t)) \\
& -\left(\int_{-\infty}^{t} \mathrm{MK}(t-s) x(s) \mathrm{d} s\right)^{T} \\
& \times \mathrm{R}\left(\int_{-\infty}^{t} \mathrm{MK}(t-s) x(s) \mathrm{d} s\right) .
\end{aligned}
$$

By $0 \leq \dot{\tau}(t) \leq h$, it is easy to derive that

$$
\begin{aligned}
\dot{V}_{4}(t)= & (\operatorname{Mf}(x(t)))^{T} \mathrm{G}(\operatorname{Mf}(x(t))) \\
& -(1-\dot{\tau}(t))(\operatorname{Mf}(x(t-\tau(t))))^{T} \\
& \times \mathrm{G}(\operatorname{Mf}(x(t-\tau(t)))) \\
\leq & (\operatorname{Mf}(x(t)))^{T} \mathrm{G}(\operatorname{Mf}(x(t))) \\
& -(1-h)(\operatorname{Mf}(x(t-\tau(t))))^{T} \\
& \times \mathrm{G}(\operatorname{Mf}(x(t-\tau(t)))), \\
\dot{V}_{5}(t) \leq & (\operatorname{Mx}(t))^{T} \mathrm{~S}(\operatorname{Mx}(t)) \\
& -(1-h)(\operatorname{Mx}(t-\tau(t)))^{T} \mathrm{~S}(\operatorname{Mx}(t-\tau(t))) .
\end{aligned}
$$

In view of assumption $\left(\mathrm{H}_{2}\right)$, for any positive diagonal matrices $S_{1}$ and $S_{2}$, the following two inequalities hold:

$$
\begin{gathered}
\left(\begin{array}{c}
\mathrm{M} x(t) \\
\mathrm{Mf}(x(t))
\end{array}\right)^{T}\left(\begin{array}{cc}
\mathrm{E}_{1} S_{1} & -\mathrm{E}_{2} S_{1} \\
-\mathrm{E}_{2}^{T} S_{1} & I_{(N-1) n} S_{1}
\end{array}\right)\left(\begin{array}{c}
\mathrm{M} x(t) \\
\mathrm{Mf}(x(t))
\end{array}\right) \leq 0, \\
\left(\begin{array}{c}
\mathrm{M} x(t-\tau(t)) \\
\mathrm{Mf}(x(t-\tau(t)))
\end{array}\right)^{T}\left(\begin{array}{cc}
\mathrm{E}_{1} S_{2} & -\mathrm{E}_{2} S_{2} \\
-\mathrm{E}_{2}^{T} S_{2} & I_{(N-1) n} S_{2}
\end{array}\right) \\
\times\left(\begin{array}{c}
\mathrm{M} x(t-\tau(t)) \\
\operatorname{Mf}(x(t-\tau(t)))
\end{array}\right) \leq 0 .
\end{gathered}
$$


Combining (13)-(20) gives

$$
\begin{aligned}
& \dot{V}(t) \leq x^{T}(t) \mathrm{M}^{T}\left(-\overline{\mathrm{C}}_{1}^{\mathrm{T}} \mathrm{P}-\mathrm{P}_{1}+\mathrm{P} \widetilde{\mathrm{U}}+\widetilde{\mathrm{U}}^{T} \mathrm{P}\right. \\
& \left.+k^{2} \mathrm{~K}^{T}(0) \mathrm{RK}(0)+\mathrm{S}-\mathrm{E}_{1}\right) \mathrm{M} x(t) \\
& +2 x^{T}(t) \mathrm{M}^{T}\left(\mathrm{PA}_{1}+\mathrm{E}_{2}\right) \mathrm{Mf}(x(t)) \\
& +2 x^{T}(t) \mathrm{M}^{T} \mathrm{~PB}_{1} \mathrm{Mf}(x(t-\tau(t))) \\
& +2 x^{T}(t) \mathrm{M}^{T} \mathrm{PD}_{1} \mathrm{M} \int_{-\infty}^{t} \mathrm{~K}(t-s) \mathrm{f}(x(s)) \mathrm{d} s \\
& +2 x^{T}(t) \mathrm{M}^{T}\left(\mathrm{P} \widetilde{\mathrm{V}}-\mathrm{P} \bar{\Upsilon}_{1}\right) \mathrm{M} x(t-\tau(t)) \\
& +2 x^{T}(t) \mathrm{M}^{T}\left(\mathrm{P} \widetilde{\mathrm{W}}-\mathrm{P} \bar{\Lambda}_{1}\right) \mathrm{M} \int_{-\infty}^{t} \mathrm{~K}(t-s) x(s) \mathrm{d} s \\
& +(\operatorname{Mf}(x(t)))^{T}\left(k^{2} \mathrm{~K}^{T}(0) \mathrm{QK}(0)+\mathrm{G}-I_{(N-1) n}\right) \\
& \times(\operatorname{Mf}(x(t))) \\
& -\left(\int_{-\infty}^{t} \operatorname{MK}(t-s) \mathrm{f}(x(s)) \mathrm{d} s\right)^{T} \\
& \times \mathrm{Q}\left(\int_{-\infty}^{t} \mathrm{MK}(t-s) \mathrm{f}(x(s)) \mathrm{d} s\right) \\
& -\left(\int_{-\infty}^{t} \operatorname{MK}(t-s) x(s) \mathrm{d} s\right)^{T} \\
& \times \mathrm{R}\left(\int_{-\infty}^{t} \mathrm{MK}(t-s) x(s) \mathrm{d} s\right) \\
& +(\operatorname{Mf}(x(t-\tau(t))))^{T}\left[-(1-h) \mathrm{G}-I_{(N-1) n}\right] \\
& \times(\operatorname{Mf}(x(t-\tau(t)))) \\
& +(\mathrm{M} x(t-\tau(t)))^{T}\left[-(1-h) \mathrm{S}-\mathrm{E}_{1}\right] \mathrm{M} x(t-\tau(t)) \\
& +(\mathrm{M} x(t-\tau(t)))^{T} \mathrm{E}_{2} \mathrm{M} f(x(t-\tau(t))) \\
& =\xi^{T} \Omega \xi \text {, }
\end{aligned}
$$

where

$$
\begin{aligned}
\xi=( & (\mathrm{M} x(t))^{T},(\mathrm{M} x(t-\tau(t)))^{T}, \\
& (\mathrm{Mf}(x(t)))^{T},(\mathrm{Mf}(x(t-\tau(t))))^{T}, \\
& \left(\mathrm{M} \int_{-\infty}^{t} \mathrm{~K}(t-s) x(s) \mathrm{d} s\right)^{T}, \\
& \left.\left(\mathrm{M} \int_{-\infty}^{t} \mathrm{~K}(t-s) \mathrm{f}(x(s)) \mathrm{d} s\right)^{T}\right)^{T} .
\end{aligned}
$$

From the given condition (12) and the inequality (21), one derives that $\dot{V}(t) \leq 0$ and $\dot{V}(t)=0$ if and only if $\xi=0$. Hence, $\lim _{t \rightarrow \infty}\left\|\left(M \otimes I_{n}\right) x(t)\right\|=0$. By virtue of Definition 5 and Lemma 8 , the coupled neural network (11) is globally asymptotically synchronize. This completes the proof.

Corresponding to (5), we now consider the following network with time-varying delays and bounded distributed delays:

$$
\begin{aligned}
\dot{x}(t)= & -\overline{\mathrm{C}} x(t)+\mathrm{Af}(x(t))+\mathrm{Bf}(x(t-\tau(t))) \\
& +\mathrm{D} \int_{t-\theta(t)}^{t} \mathrm{~K}(t-s) \mathrm{f}(x(s)) \mathrm{d} s+\mathrm{I}(t)
\end{aligned}
$$

$$
\begin{aligned}
& +\overline{\mathrm{U}} x(t)+\overline{\mathrm{V}} x(t-\tau(t)) \\
& +\overline{\mathrm{W}} \int_{t-\theta(t)}^{t} \mathrm{~K}(t-s) x(s) \mathrm{d} s-\bar{\Upsilon} x(t-\tau(t)) \\
& -\bar{\Lambda} \int_{t-\theta(t)}^{t} \mathrm{~K}(t-s) x(s) \mathrm{d} s
\end{aligned}
$$

For the system (23) the following result can be easily derived by similar proof process of Theorem 10 .

Corollary 11. Under assumptions $\left(\mathrm{H}_{2}\right)$ and $\left(\mathrm{H}_{3}\right)$, if there is positive constant $k$ such that $\int_{0}^{\theta(t)} K(u) d u=k(t) \leq k$, matrices $M \in M_{2}$ and $J$ satisfying $M J=I_{N-1}$, positive definite matrices $P, Q, R, G, S \in \mathbb{R}^{(N-1) n \times(N-1) n}$, and two positive diagonal matrices $S_{1}, S_{2} \in \mathbb{R}^{(N-1) n \times(N-1) n}$ such that the linear matrix inequality (12) holds, then the coupled neural network (23) is globally asymptotically synchronized.

Proof. Consider the following Lyapunov function:

$$
V(t)=\sum_{i=1}^{5} V_{i}(t),
$$

where $V_{1}(t), V_{4}(t)$, and $V_{5}(t)$ are the same as those defined in the proof of Theorem 10 and

$$
\begin{aligned}
& V_{2}(t)=k \int_{-\theta}^{0} \int_{t+s}^{t}(\operatorname{MK}(t-\theta) \mathrm{f}(x(\theta)))^{T} \\
& \times \mathrm{Q}(\operatorname{MK}(t-\theta) \mathrm{f}(x(\theta))) \mathrm{d} \theta \mathrm{d} s, \\
& V_{3}(t)=k \int_{-\theta}^{0} \int_{t+s}^{t}(\operatorname{MK}(t-\theta) x(\theta))^{T} \\
& \times \mathrm{R}(\operatorname{MK}(t-\theta) x(\theta)) \mathrm{d} \theta \mathrm{d} s .
\end{aligned}
$$

Based on Lemma 9, one can get that

$$
\begin{aligned}
& \dot{V}_{2}(t) \leq k^{2}(\mathrm{MK}(0) \mathrm{f}(x(t)))^{T} \mathrm{Q}(\mathrm{MK}(0) \mathrm{f}(x(t))) \\
&- k(t) \int_{t-\theta(t)}^{t}(\mathrm{MK}(t-s) \mathrm{f}(x(s)))^{T} \\
& \times \mathrm{Q}(\mathrm{MK}(t-s) \mathrm{f}(x(s))) \mathrm{d} s \\
& \leq k^{2}(\mathrm{Mf}(x(t)))^{T} \mathrm{~K}^{T}(0) \mathrm{QK}(0)(\mathrm{Mf}(x(t))) \\
&-\left(\int_{t-\theta(t)}^{t} \mathrm{MK}(t-s) \mathrm{f}(x(s)) \mathrm{d} s\right)^{T} \\
& \times \mathrm{Q}\left(\int_{t-\theta(t)}^{t} \mathrm{MK}(t-s) \mathrm{f}(x(s)) \mathrm{d} s\right), \\
& \dot{V}_{3}(t) \leq k^{2}(\mathrm{M} x(t))^{T} \mathrm{~K}^{T}(0) \mathrm{RK}(0)(\mathrm{M} x(t)) \\
&-\left(\int_{t-\theta(t)}^{t} \mathrm{MK}^{t}(t-s) x(s) \mathrm{d} s\right)^{T} \\
& \times \mathrm{R}\left(\int_{t-\theta(t)}^{t} \mathrm{MK}(t-s) x(s) \mathrm{d} s\right) .
\end{aligned}
$$

The rest part of the proof is similar to that of the proof of Theorem 10. This completes the proof. 
Remark 12. In this paper, the least restriction is imposed on the time-varying delay. The derivative of the time-varying delay can be any given value, and the time-varying delay can be unbounded. However, most of former results are based on either that the derivative of the time-varying delay should be less than $1[16,17]$ or that the time-varying delay should be bounded [16] or even both of them [10]. In this sense, results of this paper are less conservative than those of $[10,16,17]$.

Remark 13. Synchronization criteria in an array of coupled neural networks with limited transmission efficiency are obtained in Theorem 10 and Corollary 11. One may note that assumption condition $\left(\mathrm{H}_{3}\right)$ is strong. Many real-world complex dynamical network models do not satisfy $\left(\mathrm{H}_{3}\right)$ and exhibit more complicated dynamical behaviors. How to control complex networks with arbitrary limited transmission efficiency while without $\left(\mathrm{H}_{3}\right)$ is our next research topic, which is also a challenging work.

\section{Numerical Example}

In this section, one example is provided to illustrate the effectiveness of the results obtained above.

Consider a 2-dimensional neural network with both discrete and unbounded distributed delays as follows:

$$
\begin{aligned}
\dot{x}(t)= & -C x(t)+A f(x(t))+B f\left(x_{i}(t-\tau(t))\right) \\
& +D \int_{-\infty}^{t} K(t-s) f(x(s)) d s+I(t),
\end{aligned}
$$

where $x(t)=\left(x_{1}(t), x_{2}(t)\right)^{T}, f(x(t))=\left(\tanh \left(x_{1}(t)\right)\right.$, $\left.\tanh \left(x_{2}(t)\right)\right)^{T}, \tau(t)=1, k(t)=e^{-0.5 t}$, and

$$
\begin{gathered}
C=\left(\begin{array}{cc}
1.2 & 0 \\
0 & 1
\end{array}\right), \quad A=\left(\begin{array}{cc}
3 & -0.3 \\
4 & 5
\end{array}\right), \\
B=\left(\begin{array}{cc}
-1.4 & 0.1 \\
0.3 & -8
\end{array}\right), \quad D=\left(\begin{array}{cc}
-1.2 & 0.1 \\
-2.8 & -1
\end{array}\right), \\
I(t)=\left(\begin{array}{c}
1 \\
1.2
\end{array}\right) .
\end{gathered}
$$

In the case that the initial condition is chosen as $x(t)=$ $(0.4,0.6)^{T}, \forall t \in[-1,0]$, and $x(t)=0$ for $t<-1$, the chaoticlike trajectory of (28) can be seen in Figure 1.

Now we consider a coupled neural network consisting of five identical models (28), which is described as

$$
\begin{aligned}
\dot{x}_{i}(t)= & -C x_{i}(t)+A f\left(x_{i}(t)\right)+B f\left(x_{i}(t-\tau(t))\right) \\
& +D \int_{-\infty}^{t} K(t-s) f\left(x_{i}(s)\right) d s+I(t) \\
& +\sum_{j=1}^{N} \alpha_{i j} u_{i j} \Phi\left(x_{j}(t)-x_{i}(t)\right) \\
& +\sum_{j=1}^{N} \beta_{i j} v_{i j} \Upsilon\left(x_{j}(t-\tau(t))-x_{i}(t-\tau(t))\right)
\end{aligned}
$$

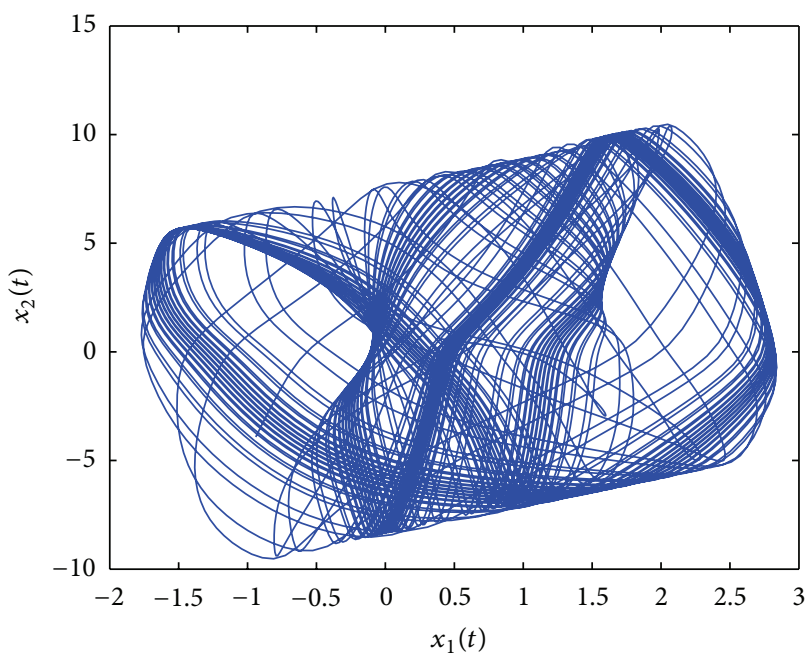

FIGURE 1: Chaotic-like trajectory of the system (28).

$$
\begin{array}{r}
+\sum_{j=1}^{N} \gamma_{i j} w_{i j} \Lambda \int_{-\infty}^{t} K(t-s)\left(x_{j}(s)-x_{i}(s)\right) d s \\
i=1,2, \ldots, 5
\end{array}
$$

where $x_{i}(t)=\left(x_{i 1}(t), x_{i 2}(t)\right)^{T}$ is the state of the $i$ th neural network, $\Phi, \Upsilon$, and $\Lambda$ are identity matrices, $U, V$, and $W$ are asymmetric and zero-row sum matrices as the following:

$$
\begin{aligned}
& U=10\left(\begin{array}{ccccc}
-7 & 1 & 3 & 2 & 1 \\
1 & -4 & 1 & 0 & 2 \\
1 & 0 & -3 & 1 & 1 \\
1 & 1 & 1 & -4 & 1 \\
2 & 0 & 2 & 1 & -5
\end{array}\right), \\
& V=W=\left(\begin{array}{ccccc}
-3 & 0 & 1 & 1 & 1 \\
0 & -2 & 1 & 0 & 1 \\
0 & 1 & -2 & 0 & 1 \\
1 & 0 & 0 & -1 & 0 \\
0 & 1 & 0 & 1 & -2
\end{array}\right),
\end{aligned}
$$

the transmission efficiency matrices are

$$
\begin{gathered}
\alpha=\left(\begin{array}{ccccc}
1 & 0.99 & 1 & 1 & 0.99 \\
1 & 1 & 1 & 0 & 0.99 \\
0.98 & 0 & 1 & 1 & 1 \\
1 & 0.99 & 0.99 & 1 & 1 \\
1 & 0 & 1 & 0.98 & 1
\end{array}\right), \\
\beta=\Gamma=\left(\begin{array}{ccccc}
1 & 0 & 0.9 & 0.9 & 0.9 \\
0 & 1 & 0.9 & 0 & 0.8 \\
0 & 0.8 & 1 & 0 & 0.9 \\
0.7 & 0 & 0 & 1 & 0 \\
0 & 0.9 & 0 & 0.8 & 1
\end{array}\right) .
\end{gathered}
$$

It is easy to check that the activation function $f$ satisfies assumption $\left(\mathrm{H}_{2}\right)$, and $\widehat{E}_{1}=0, \widehat{E}_{2}=\operatorname{diag}(0.5,0.5)$. Moreover, 


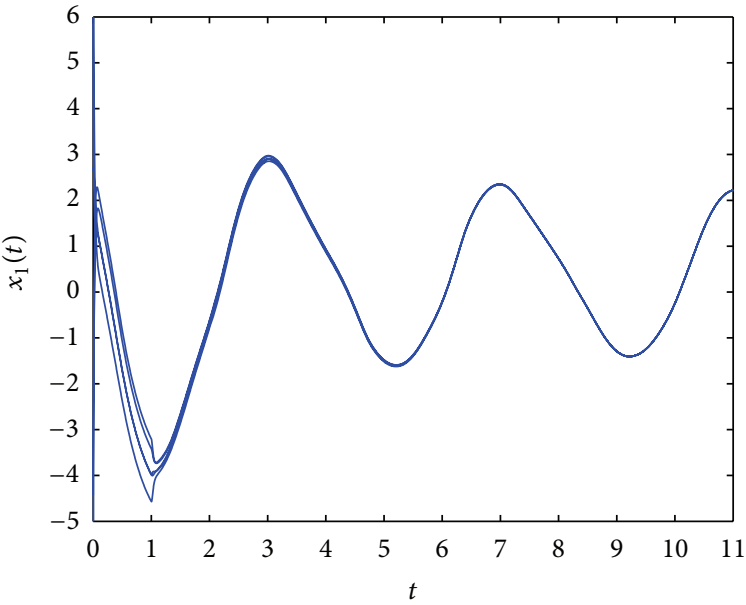

(a)

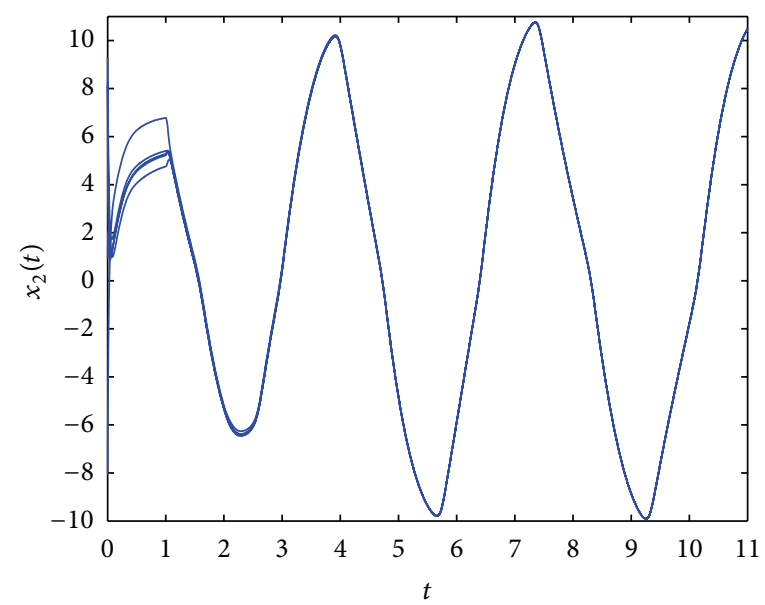

(b)

FIGURE 2: Time response of $x_{i 1}(t)$ (a) and $x_{i 2}(t)(\mathrm{b}), i=1,2, \ldots, 5$.

$\left(\mathrm{H}_{1}\right)$ and $\left(\mathrm{H}_{3}\right)$ are satisfied with $k=2, a=0.2$, and $b=c=$ 0.3 . Obviously, $h=0$. Take

$$
\begin{gathered}
M=\left(\begin{array}{ccccc}
1 & -1 & 0 & 0 & 0 \\
0 & 1 & -1 & 0 & 0 \\
0 & 0 & 1 & -1 & 0 \\
0 & 0 & 0 & 1 & -1
\end{array}\right), \\
J=\left(\begin{array}{llll}
1 & 1 & 1 & 1 \\
0 & 1 & 1 & 1 \\
0 & 0 & 1 & 1 \\
0 & 0 & 0 & 1 \\
0 & 0 & 0 & 0
\end{array}\right)
\end{gathered}
$$

According to Theorem 10, by referring to the MATLAB LMI Toolbox, one can get the feasible solution, see the appendix at the end of this paper. Hence, the system (30) is globally asymptotically synchronized.

In the simulations, the Runge-Kutta numerical scheme is used to simulate by MATLAB. The initial values of (30) are chosen randomly in the real number interval $[-10,10]$ for $t \in[-1,0]$ and all the states of the coupled neural networks are zero for $t<0$. The time step size is $\delta=0.005$. Figure 2 shows the time response of the states. Figure 3 describes the synchronization errors $e(t)=\sum_{j=1}^{2} \sqrt{\sum_{i=2}^{5}\left[x_{1 j}-x_{i j}\right]^{2}}$, which turn to zero quickly as time goes.

Figure 4 presents the synchronized state of (30), which is different from that of Figure 1. Actually, it can be seen from (8) that $a, b$, and $c$ and $\Phi, \Upsilon$, and $\Lambda$ have important effects on the synchronized state. Let

$$
\Phi=\left(\begin{array}{cc}
1 & 0 \\
0.5 & 1
\end{array}\right), \quad \Upsilon=\left(\begin{array}{cc}
0.5 & 0 \\
1 & 1
\end{array}\right), \quad \Lambda=\left(\begin{array}{cc}
0.5 & 0 \\
0 & 0.5
\end{array}\right)
$$

in (8). Figure 5 depicts the trajectories of (8) with different $a$, $b$, and $c$, the other parameters are the same as those in (28).

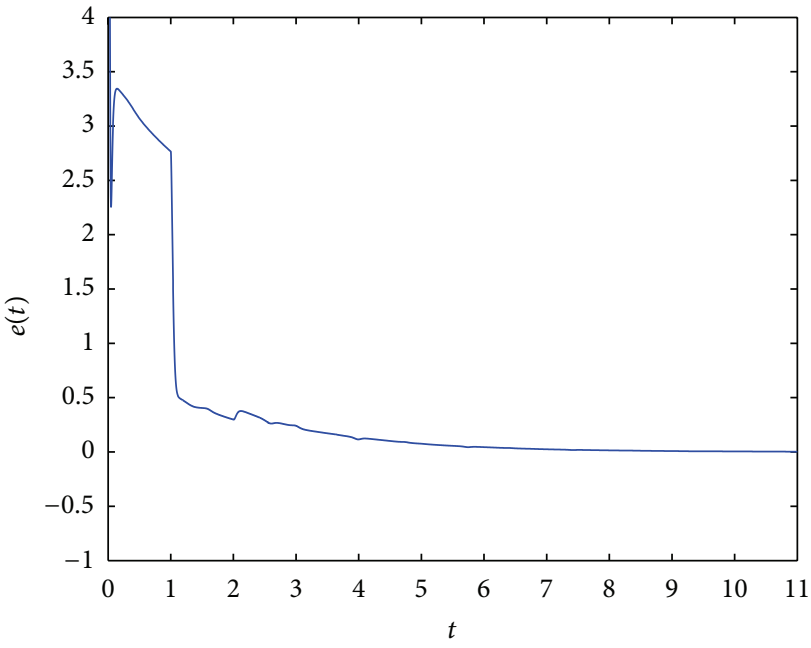

FIGURE 3: Error distance of the coupled network (30).

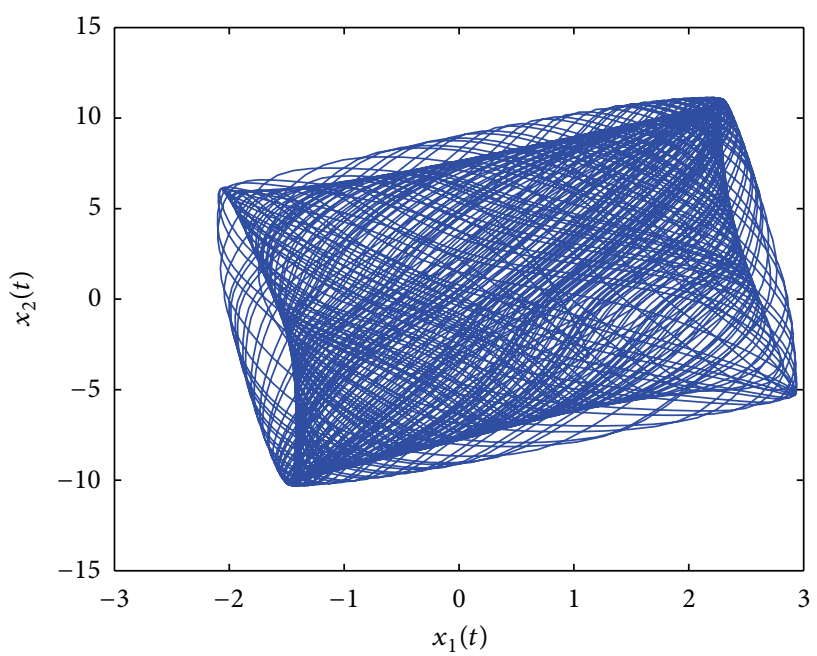

FIGURE 4: Trajectory of the synchronized state of system (30). 


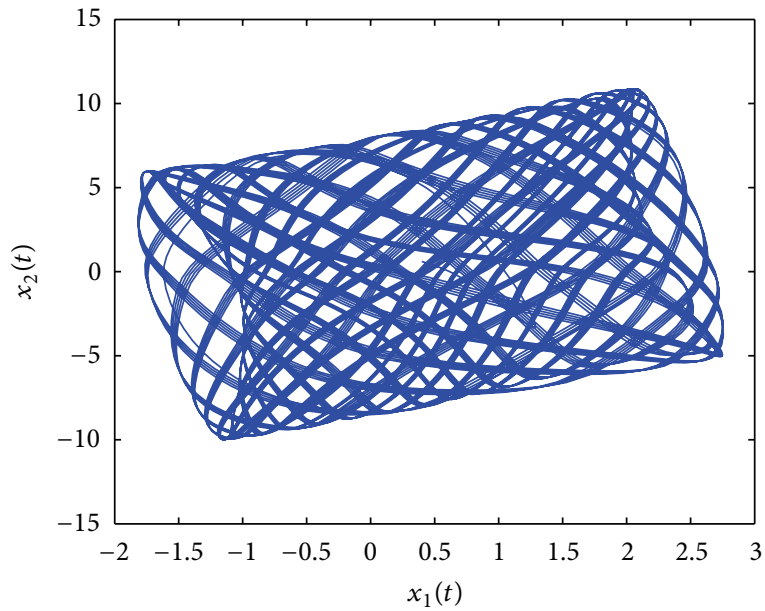

(a)

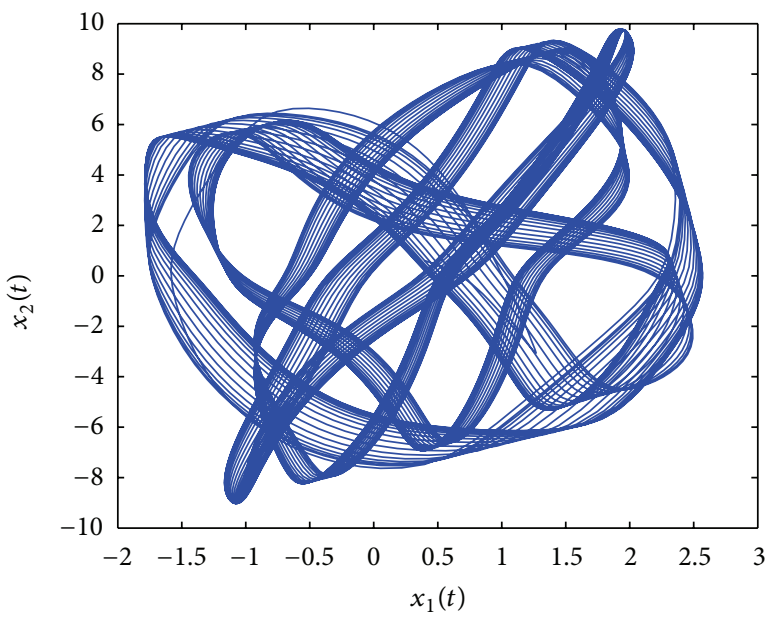

(c)

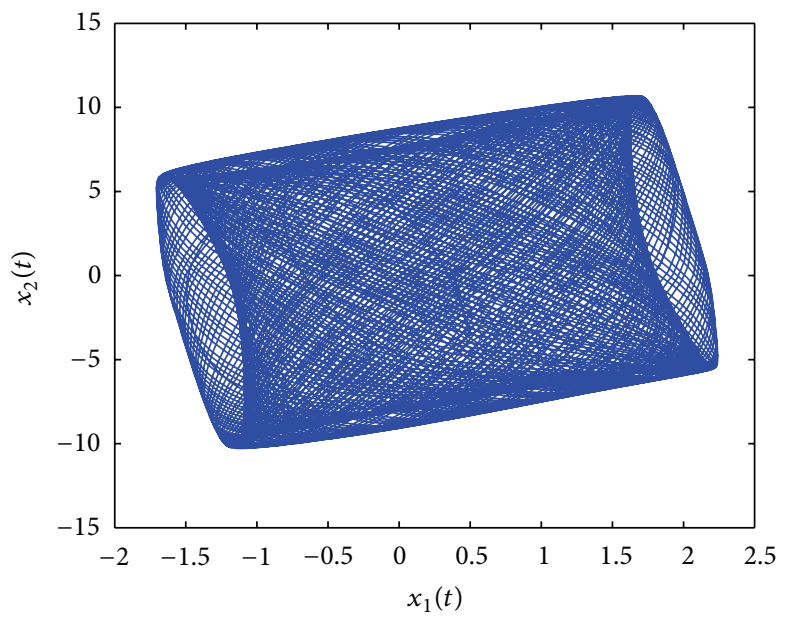

(e)

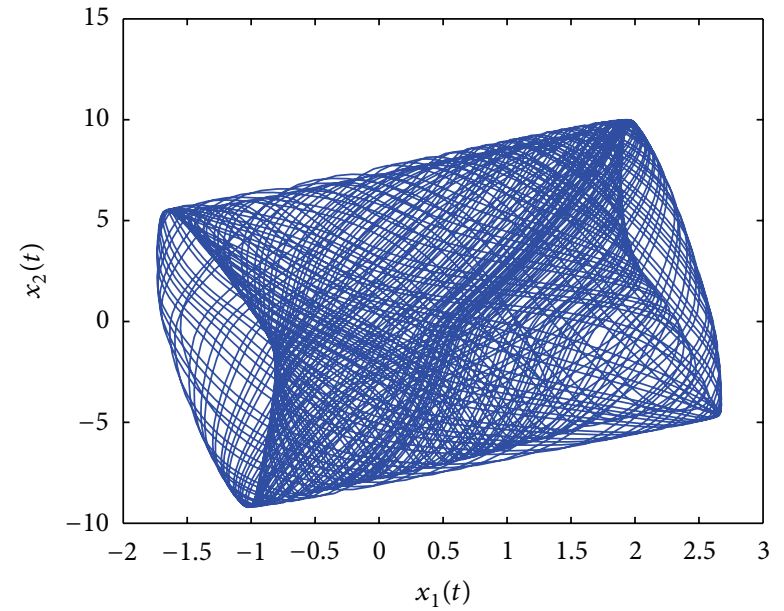

(b)

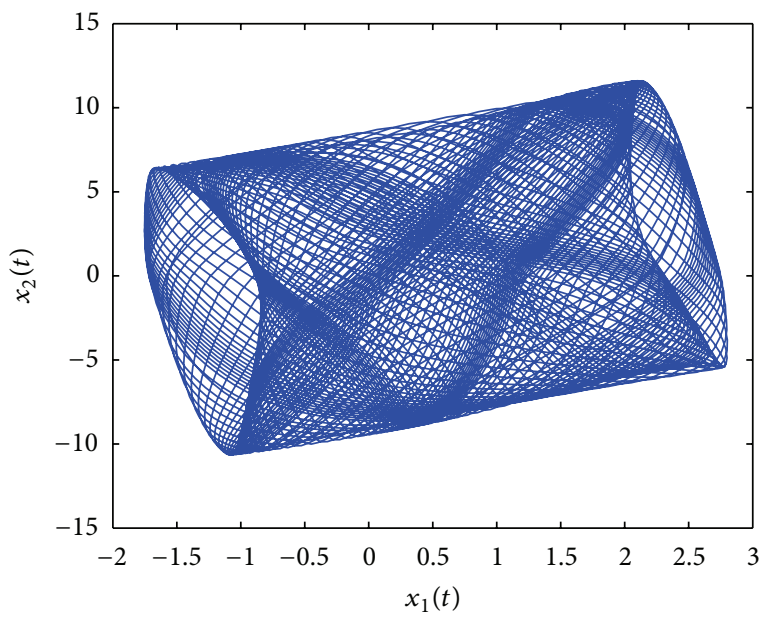

(d)

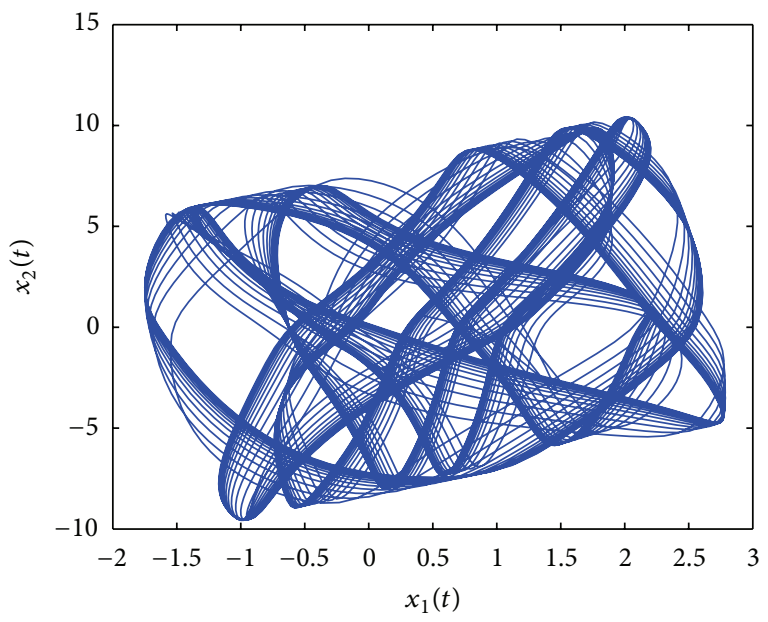

(f)

FIGURE 5: Trajectories of system (8) with different $a, b$, and $c$ : (a) $a=0.2, b=0.3$, and $c=0.3$; (b) $a=0.2, b=0.1$, and $c=0.3$; (c) $a=0.2$, $b=0$, and $c=0.5$; (d) $a=0.2, b=0.5$, and $c=0$; (e) $a=1, b=1$, and $c=1$; (f) $a=0.1, b=0.1$, and $c=0.1$. 


\section{Conclusions}

In this paper, a general model of coupled neural networks with time-varying delays and unbounded distributed delays is proposed. Limited transmission efficiency between coupled nodes is considered in the dynamical network model. Based on the integral inequality and the Lyapunov functional method, sufficient conditions in terms of LMIs are derived to guarantee the synchronization of the proposed dynamical network with limited transmission efficiency. The restriction on time-varying delay is the least. The derivative of the time-varying delay can be any given value, and the timevarying delay can be unbounded. Numerical examples are given to verify the effectiveness of the theoretical results. Furthermore, numerical simulations show that, when some of the transmission efficiencies are less than 1, the transmission efficiency and inner coupling matrices between nodes play important roles for the final synchronized state. Since many real-world transmission efficiencies between nodes are usually less than 1 , the results of this paper are new and extend some of the existing results.

\section{Appendix}

$$
\begin{aligned}
& P=\left(\begin{array}{cccccccc}
2.3293 & -0.0507 & -0.4456 & 0.0497 & -0.1796 & 0.0081 & -0.0216 & -0.0071 \\
-0.0507 & 2.0002 & 0.0479 & -0.1233 & 0.0083 & -0.1427 & -0.0066 & -0.0497 \\
-0.4456 & 0.0479 & 4.9740 & -0.1330 & -0.1868 & 0.0201 & -0.4820 & 0.0022 \\
0.0497 & -0.1233 & -0.1330 & 3.8409 & 0.0208 & -0.0304 & 0.0009 & -0.4124 \\
-0.1796 & 0.0083 & -0.1868 & 0.0208 & 3.8034 & -0.0981 & -0.2548 & 0.0138 \\
0.0081 & -0.1427 & 0.0201 & -0.0304 & -0.0981 & 3.0951 & 0.0139 & -0.1496 \\
-0.0216 & -0.0066 & -0.4820 & 0.0009 & -0.2548 & 0.0139 & 3.1954 & -0.0674 \\
-0.0071 & -0.0497 & 0.0022 & -0.4124 & 0.0138 & -0.1496 & -0.0674 & 2.7416
\end{array}\right) \\
& Q=\left(\begin{array}{cccccccc}
14.1261 & -0.3969 & 0.1779 & 0.0687 & -0.0216 & 0.0133 & 0.0373 & 0.0077 \\
-0.3969 & 12.1352 & 0.0901 & 0.7797 & 0.0090 & 0.0242 & 0.0046 & 0.0984 \\
0.1779 & 0.0901 & 13.7184 & -0.5675 & 0.1003 & 0.0460 & 0.1842 & 0.0075 \\
0.0687 & 0.7797 & -0.5675 & 9.3374 & 0.0397 & 0.4060 & 0.0101 & 0.4532 \\
-0.0216 & 0.0090 & 0.1003 & 0.0397 & 14.0715 & -0.5518 & 0.0850 & 0.0348 \\
0.0133 & 0.0242 & 0.0460 & 0.4060 & -0.5518 & 10.8553 & 0.0308 & 0.3573 \\
0.0373 & 0.0046 & 0.1842 & 0.0101 & 0.0850 & 0.0308 & 14.1750 & -0.4777 \\
0.0077 & 0.0984 & 0.0075 & 0.4532 & 0.0348 & 0.3573 & -0.4777 & 11.7793
\end{array}\right), \\
& R=\left(\begin{array}{cccccccc}
51.1311 & -2.8226 & 2.1854 & 0.6937 & -0.6251 & 0.2521 & 0.7007 & -0.0726 \\
-2.8226 & 36.5803 & 0.6786 & 7.1163 & 0.2622 & 0.2684 & -0.0770 & 0.6599 \\
2.1854 & 0.6786 & 44.5831 & -3.7741 & 1.5536 & 0.2002 & 1.6747 & 0.0327 \\
0.6937 & 7.1163 & -3.7741 & 22.4396 & 0.2177 & 3.3233 & 0.0238 & 1.9847 \\
-0.6251 & 0.2622 & 1.5536 & 0.2177 & 49.0212 & -3.7531 & 1.3611 & 0.2250 \\
0.2521 & 0.2684 & 0.2002 & 3.3233 & -3.7531 & 28.4807 & 0.2336 & 3.0901 \\
0.7007 & -0.0770 & 1.6747 & 0.0238 & 1.3611 & 0.2336 & 50.7054 & -3.3712 \\
-0.0726 & 0.6599 & 0.0327 & 1.9847 & 0.2250 & 3.0901 & -3.3712 & 33.8828
\end{array}\right), \\
& G=\left(\begin{array}{cccccccc}
-31.8299 & -0.6083 & 0.0156 & 0.0565 & 0.2454 & 0.0531 & -0.0814 & 0.0392 \\
-0.6083 & -26.2338 & 0.1191 & -2.0217 & 0.0520 & 0.0315 & 0.0325 & -0.3113 \\
0.0156 & 0.1191 & -33.6328 & -1.6051 & -0.0230 & 0.0458 & -0.0143 & 0.1821 \\
0.0565 & -2.0217 & -1.6051 & -18.9430 & 0.0376 & -1.0840 & 0.1756 & -1.5906 \\
0.2454 & 0.0520 & -0.0230 & 0.0376 & -33.4900 & -1.2137 & 0.0722 & 0.0789 \\
0.0531 & 0.0315 & 0.0458 & -1.0840 & -1.2137 & -23.4590 & 0.0800 & -0.9480 \\
-0.0814 & 0.0325 & -0.0143 & 0.1756 & 0.0722 & 0.0800 & -33.1772 & -0.9452 \\
0.0392 & -0.3113 & 0.1821 & -1.5906 & 0.0789 & -0.9480 & -0.9452 & -26.0874
\end{array}\right) \text {, }
\end{aligned}
$$




$$
\begin{gathered}
S=\left(\begin{array}{cccccccc}
81.6408 & -0.4942 & 0.3979 & 0.5882 & 0.2149 & -0.0492 & 0.0804 & -0.0541 \\
-0.4942 & 75.1089 & 0.5176 & 5.0908 & -0.0392 & 0.5506 & -0.0392 & -0.3170 \\
0.3979 & 0.5176 & 84.7411 & -1.2587 & 0.4128 & 0.2505 & -0.1328 & 0.0971 \\
0.5882 & 5.0908 & -1.2587 & 68.4426 & 0.2667 & 2.7250 & 0.0774 & 0.7954 \\
0.2149 & -0.0392 & 0.4128 & 0.2667 & 83.2899 & -0.5940 & 0.3435 & 0.1248 \\
-0.0492 & 0.5506 & 0.2505 & 2.7250 & -0.5940 & 72.7484 & 0.1203 & 1.8855 \\
0.0804 & -0.0392 & -0.1328 & 0.0774 & 0.3435 & 0.1203 & 82.3409 & -0.3789 \\
-0.0541 & -0.3170 & 0.0971 & 0.7954 & 0.1248 & 1.8855 & -0.3789 & 74.7768
\end{array}\right), \\
S_{1}=\operatorname{diag}(63.3310,61.7187,65.1691,60.1717,64.2638,62.0351,63.7512,62.1022), \\
S_{2}=\operatorname{diag}(67.5321,66.8113,70.6801,69.0580,69.6841,69.7116,69.0126,69.7827) .
\end{gathered}
$$

\section{Acknowledgments}

This work was jointly supported by the National Natural Science Foundation of China (NSFC) under Grants nos. 61263020, 61272530, and 11072059, the Natural Science Foundation of Jiangsu Province of China under Grant no. BK2012741, the Scientific Research Fund of Yunnan Province under Grant no. 2010ZC150, and the Scientific Research Fund of Chongqing Normal University under Grants no. 12XLB031 and no. 940115.

\section{References}

[1] S. H. Strogatz and I. Stewart, "Coupled oscillators and biological synchronization," Scientific American, vol. 269, no. 6, pp. 102109, 1993.

[2] P. Zhou and R. Ding, "Modified function projective synchronization between different dimension fractional-order chaotic systems," Abstract and Applied Analysis, vol. 2012, Article ID 862989, 12 pages, 2012.

[3] X. Yang and J. Cao, "Finite-time stochastic synchronization of complex networks," Applied Mathematical Modelling, vol. 34, no. 11, pp. 3631-3641, 2010.

[4] J. Liang, Z. Wang, Y. Liu, and X. Liu, "Global synchronization control of general delayed discrete-time networks with stochastic coupling and disturbances," IEEE Transactions on Systems, Man, and Cybernetics B, vol. 38, no. 4, pp. 1073-1083, 2008.

[5] X. Yang, Q. Zhu, and C. Huang, "Lag stochastic synchronization of chaotic mixed time-delayed neural networks with uncertain parameters or perturbations," Neurocomputing, vol. 74, no. 10, pp. 1617-1625, 2011.

[6] J. Cao and J. Lu, "Adaptive synchronization of neural networks with or without time-varying delay," Chaos, vol. 16, no. 1, Article ID 013133, 6 pages, 2006.

[7] X. Yang, J. Cao, Y. Long, and W. Rui, "Adaptive lag synchronization for competitive neural networks with mixed delays and uncertain hybrid perturbations," IEEE Transactions on Neural Networks, vol. 21, no. 10, pp. 1656-1667, 2010.

[8] X. Yang and J. Cao, "Stochastic synchronization of coupled neural networks with intermittent control," Physics Letters A, vol. 373, no. 36, pp. 3259-3272, 2009.

[9] W. Yu and J. Cao, "Synchronization control of stochastic delayed neural networks," Physica A, vol. 373, pp. 252-260, 2007.

[10] X. Gao, S. Zhong, and F. Gao, "Exponential synchronization of neural networks with time-varying delays," Nonlinear Analysis:
Theory, Methods \& Applications, vol. 71, no. 5-6, pp. 2003-2011, 2009.

[11] T. Li, A. Song, S. Fei, and Y. Guo, "Synchronization control of chaotic neural networks with time-varying and distributed delays," Nonlinear Analysis: Theory, Methods \& Applications, vol. 71, no. 5-6, pp. 2372-2384, 2009.

[12] J. Lu, D. W. C. Ho, and Z. Wang, "Pinning stabilization of linearly coupled stochastic neural networks via minimum number of controllers," IEEE Transactions on Neural Networks, vol. 20, no. 10, pp. 1617-1629, 2009.

[13] X. Yang, C. Huang, and Z. Yang, "Stochastic synchronization of reaction-diffusion neural networks under general impulsive controller with mixed delays," Abstract and Applied Analysis, vol. 2012, Article ID 603535, 25 pages, 2012.

[14] J. Lu and D. W. C. Ho, "Globally exponential synchronization and synchronizability for general dynamical networks," IEEE Transactions on Systems, Man, and Cybernetics B, vol. 40, no. 2, pp. 350-361, 2010.

[15] J. Lu, D. W. C. Ho, and J. Cao, "A unified synchronization criterion for impulsive dynamical networks," Automatica, vol. 46, no. 7, pp. 1215-1221, 2010.

[16] W. He and J. Cao, "Global synchronization in arrays of coupled networks with one single time-varying delay coupling," Physics Letters A, vol. 373, no. 31, pp. 2682-2694, 2009.

[17] J. Cao, Z. Wang, and Y. Sun, "Synchronization in an array of linearly stochastically coupled networks with time delays," Physica A, vol. 385, no. 2, pp. 718-728, 2007.

[18] W. Yu, J. Cao, G. Chen, J. Lü, J. Han, and W. Wei, "Local synchronization of a complex network model," IEEE Transactions on Systems, Man, and Cybernetics B, vol. 39, no. 1, pp. 230-241, 2009.

[19] J. Cao and L. Li, "Cluster synchronization in an array of hybrid coupled neural networks with delay," Neural Networks, vol. 22, no. 4, pp. 335-342, 2009.

[20] Q. Song, "Synchronization analysis of coupled connected neural networks with mixed time delays," Neurocomputing, vol. 72, no. 16-18, pp. 3907-3914, 2009.

[21] B. Shen, Z. Wang, and X. Liu, "Bounded $\mathrm{H}_{\infty}$ synchronization and state estimation for discrete time-varying stochastic complex networks over a finite horizon," IEEE Transactions on Neural Networks, vol. 22, no. 1, pp. 145-157, 2011.

[22] H. J. Gao, J. Lam, and G. Chen, "New criteria for synchronization stability of general complex dynamical networks with coupling delays," Physics Letters A, vol. 360, no. 2, pp. 263-273, 2006. 
[23] Y. Tang, R. Qiu, J. Fang, Q. Miao, and M. Xia, "Adaptive lag synchronization in unknown stochastic chaotic neural networks with discrete and distributed time-varying delays," Physics Letters A, vol. 372, no. 24, pp. 4425-4433, 2008.

[24] Y. Liu, Z. Wang, and X. Liu, "Exponential synchronization of complex networks with Markovian jump and mixed delays," Physics Letters A, vol. 372, no. 22, pp. 3986-3998, 2008.

[25] T. Li, S. M. Fei, and K. J. Zhang, "Synchronization control of recurrent neural networks with distributed delays," Physica A, vol. 387, no. 4, pp. 982-996, 2008.

[26] K. Gopalsamy and X. Z. He, "Stability in asymmetric Hopfield nets with transmission delays," Physica D, vol. 76, no. 4, pp. 344358, 1994.

[27] C. Huang and J. Cao, "Almost sure exponential stability of stochastic cellular neural networks with unbounded distributed delays," Neurocomputing, vol. 72, no. 13-15, pp. 3352-3356, 2009.

[28] L. Sheng and H. Yang, "Exponential synchronization of a class of neural networks with mixed time-varying delays and impulsive effects," Neurocomputing, vol. 71, no. 16-18, pp. 3666-3674, 2008.

[29] X. Nie and J. Cao, "Multistability of competitive neural networks with time-varying and distributed delays," Nonlinear Analysis: Real World Applications, vol. 10, no. 2, pp. 928-942, 2009.

[30] X. Yang, "Existence and global exponential stability of periodic solution for Cohen-Grossberg shunting inhibitory cellular neural networks with delays and impulses," Neurocomputing, vol. 72, no. 10-12, pp. 2219-2226, 2009.

[31] Z. Wang, Y. Liu, M. Li, and X. Liu, "Stability analysis for stochastic Cohen-Grossberg neural networks with mixed time delays," IEEE Transactions on Neural Networks, vol. 17, no. 3, pp. 814820, 2006.

[32] R. A. Horn and C. R. Johnson, Matrix Analysis, Cambridge University Press, 1990.

[33] Z. H. Guan, Z. W. Liu, G. Feng, and Y. W. Wang, "Synchronization of complex dynamical networks with time-varying delays via impulsive distributed control," IEEE Transactions on Circuits and Systems I, vol. 57, no. 8, pp. 2182-2195, 2010.

[34] Y. Wang, L. Xie, and C. E. de Souza, "Robust control of a class of uncertain nonlinear systems," Systems \& Control Letters, vol. 19, no. 2, pp. 139-149, 1992.

[35] X. Yang, J. Cao, and J. Lu, "Synchronization of coupled neural networks with random coupling strengths and mixed probabilistic time-varying delays," International Journal of Robust and Nonlinear Control, 2012. 


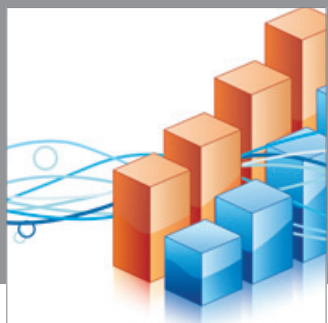

Advances in

Operations Research

mansans

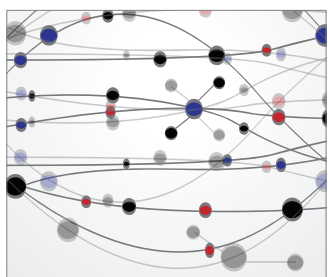

The Scientific World Journal
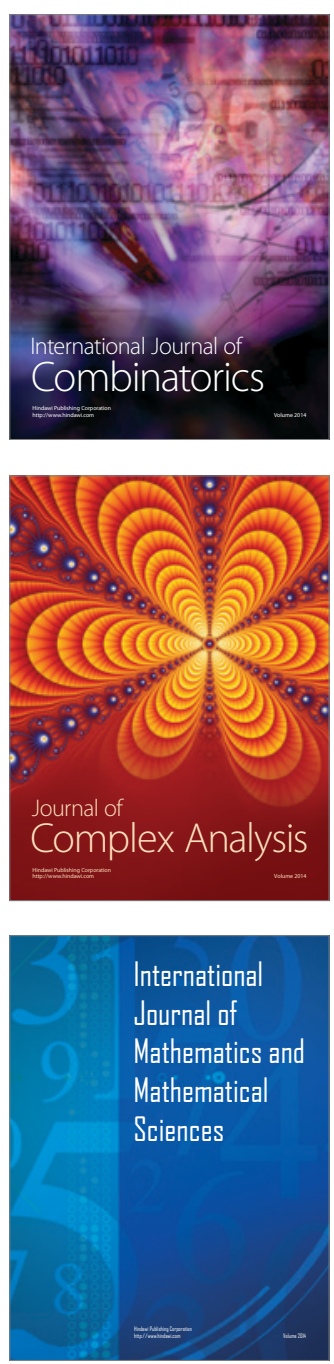
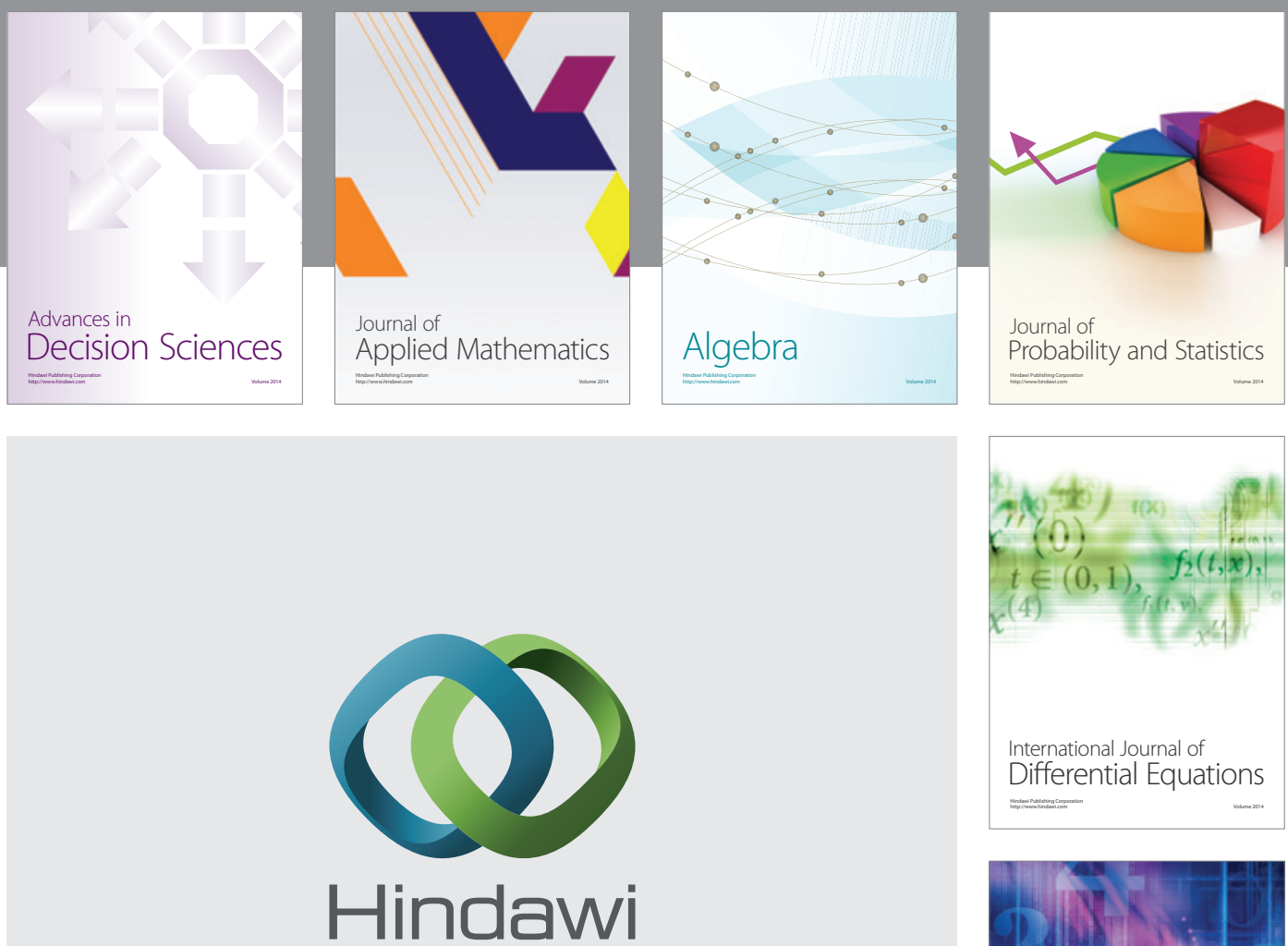

Submit your manuscripts at http://www.hindawi.com
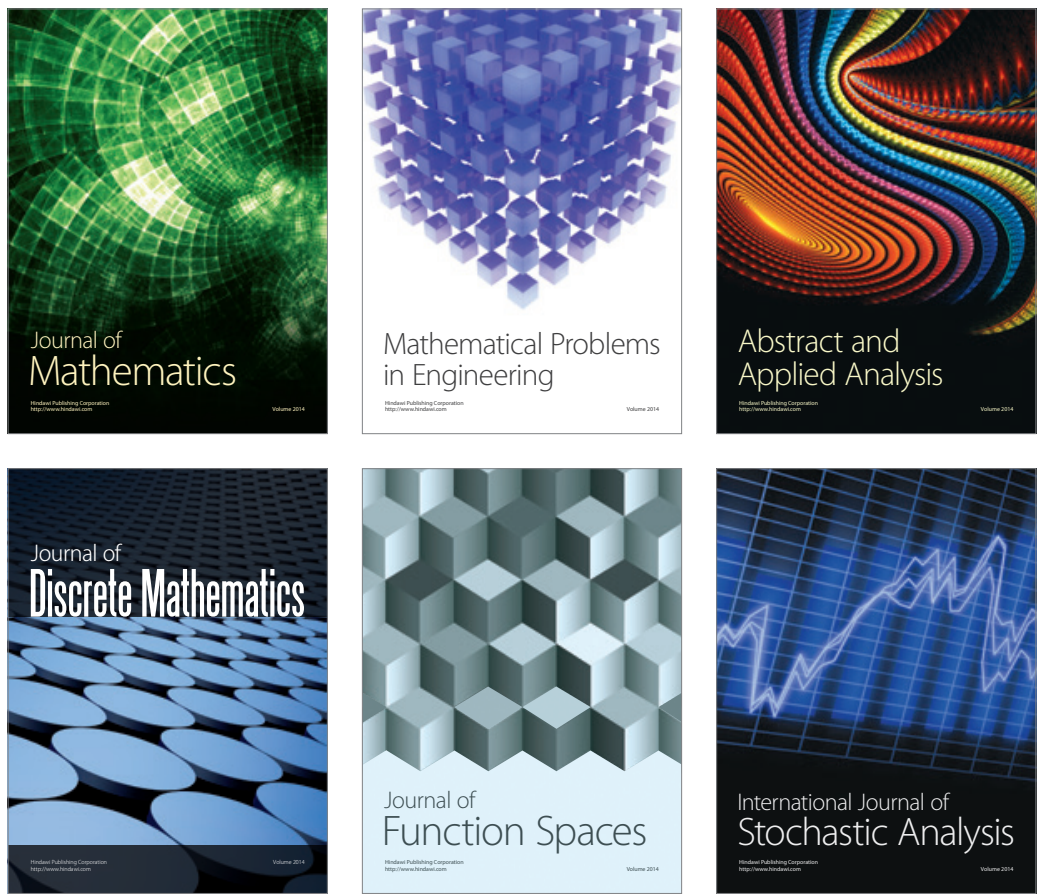

Journal of

Function Spaces

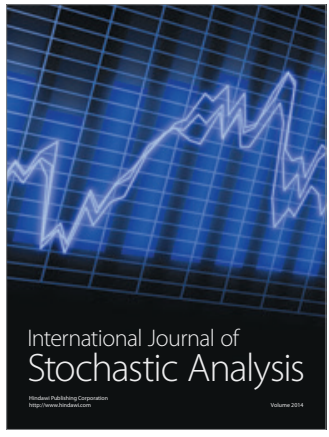

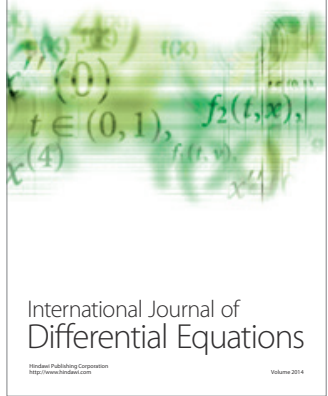
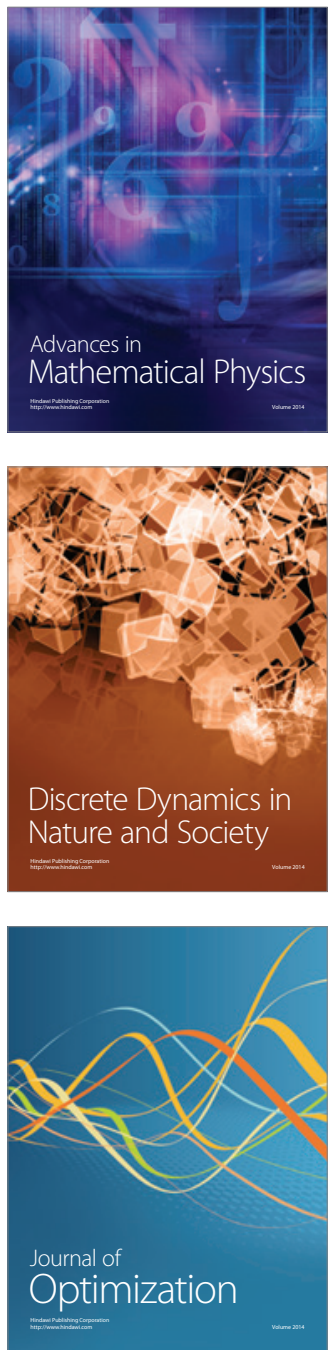